\title{
IMPRESIÓN 3D COMO UN RECURSO PARA DESARROLLAR EL POTENCIAL MATEMÁTICO
}

\author{
Álvaro Nolla \\ Angélica Benito \\ Carlo Madonna \\ Seong Suk Park \\ Universidad Autónoma de Madrid \\ Marco Busatto \\ Universidad Carlos III
}

\begin{abstract}
RESUMEN: Este trabajo recoge las experiencias con el uso de la impresión 3D en un club de matemáticas de Madrid que se llevaron a cabo entre los años 2017 y 2019. Tras el análisis de las producciones de los alumnos se distinguen cuatro tipos de proyectos que se pueden trabajar en un aula de matemáticas con el apoyo de la impresión 3D que fomentaron el trabajo colaborativo.
\end{abstract}

PALABRAS CLAVE: clubes de matemáticas, impresión 3D educativa, talento matemático.

\section{D PRINTING AS AN EDUCATIONAL RESOURCE TO DEVELOP MATHEMATICAL TALENT}

\begin{abstract}
This article contains the experiences in the use of 3D printing in a math club at Madrid which took place between 2017 and 2019. After the analysis of students' productions we distinguish four types of projects that can be included in a math classroom using a 3D printer that encouraged collaborative work.
\end{abstract}

KEYWORDS: Math clubs, educational 3D printing, math talent.

Recibido: 30/01/2021

Aceptado: 01/05/2021

Correspondencia: Álvaro Nolla de Celis, Facultad de Formación del Profesorado y Educación, Universidad Autónoma de Madrid, C/ Francisco Tomás y Valiente, 3, 28049 Madrid. Email: alvaro.nolla@uam.es 


\section{INTRODUCCIÓN}

Los clubes de matemáticas en las etapas de educación primaria y secundaria se han implantado con éxito en numerosos países. Podría definirse un club de matemáticas como "un conjunto de personas, estudiantes y docentes, que tienen en común el placer de hacer matemática y que se dedican a estudiar contenidos matemáticos, extracurriculares o también curriculares pero caracterizados por un nivel de dificultad muy elevado, resolviendo problemas propuestos y formulando problemas nuevos para resolverlos" (Madonna y Park, 2017, p. 12). Nacen, dicho en otras palabras, con el objetivo de divertirse haciendo matemáticas, fomentar el razonamiento lógico, y alimentar la curiosidad y el espíritu creativo de los alumnos. Estos clubes utilizan actividades de ampliación y revisión de contenidos curriculares y extracurriculares, desarrollando las capacidades de análisis, resolución y formulación de problemas matemáticos no estándares en una atmósfera distendida.

Este tipo de entorno educativo se presta también a desarrollar proyectos interdisciplinares, resaltando y poniendo en valor el papel que juegan las matemáticas. Estas actividades se pueden englobar dentro del enfoque STEM (Science, Technology, Engineering and Mathematics), que aboga por la enseñanza de estas disciplinas de manera integrada en lugar de áreas de conocimiento compartimentadas. Este modelo busca dar a los estudiantes la oportunidad de experimentar diferentes formas de aprender y resolver problemas interdisciplinares con una clara aplicación práctica.

Dentro de este enfoque, un ejemplo con gran potencial para un aprendizaje de las matemáticas de manera integrada con otras disciplinas es la impresión 3D. Su inclusión como herramienta educativa en un aula de matemáticas permite crear conexiones de esta disciplina con el diseño 3D, la tecnología y la ingeniería, fomentando la creatividad y mejorando las actitudes hacia las asignaturas STEM (Stansell y TylerWood, 2016). Es importante enseñar a los alumnos a que conozcan las aplicaciones y potencialidades de esta herramienta, y prepararlos para un mundo en el que este tipo de tecnología será de uso común (Department of Education, 2013).

Siendo la impresión 3D un recurso novedoso, pero con un paso firme hacia una implantación cada vez mayor en contextos educativos, tiene sentido reflexionar sobre qué tipo de actividades se pueden realizar en un aula con esta tecnología para sacar el mayor partido en su apoyo al aprendizaje de las matemáticas.

Dentro de este marco, durante los cursos 2017-2018, 2018-2019 y 2019-2020 se desarrollaron en el Club de Matemáticas de la Scuola Italiana de Madrid distintas actividades de impresión 3D con el objetivo de profundizar y aprender matemáticas desde un nuevo punto de vista. La primera de estas actividades se incluyó dentro del proyecto "Aeromodelismo y Matemáticas" donde los alumnos pudieron ver una aplicación práctica de las matemáticas en otra disciplina mediante el uso de la impresión 3D.

El trabajo con la impresora 3D continuó durante los siguientes cursos con el objetivo de afianzar y profundizar tanto en el conocimiento de las distintas fases del proceso de impresión 3D por parte de los alumnos, como de desarrollar su capacidad de modelización y visualización gracias al manejo de las herramientas tecnológicas de diseño de modelos tridimensionales.

El primer objetivo de este artículo es presentar la experiencia del Club de Matemáticas de la Scuola Italiana de Madrid con la impresión 3D y realizar un análisis y 
discusión de las producciones de los alumnos. Además, como segundo objetivo se realiza una clasificación del tipo de actividades de modelado e impresión 3D que realizaron los alumnos. Esta clasificación nos permite reflexionar sobre qué tipo de proyectos pueden hacerse en un aula que usen esta tecnología para apoyar el aprendizaje de las matemáticas.

\section{Marco teórico}

\section{La impresión 3D como herramienta educativa}

La introducción de la tecnología de impresión 3D ha supuesto una revolución en la forma de enseñar (ver Ford y Minshall (2018) para una revisión bibliográfica). No solamente dota al docente de una herramienta para diseñar material didáctico, sino que permite a los estudiantes combinar teoría y práctica. En particular, en la enseñanza de las matemáticas el potencial de esta tecnología es elevado: permite desarrollar habilidades de visualización y razonamiento espacial, integra el uso de herramientas digitales para la creación y manipulación de modelos tridimensionales, conecta de manera práctica varias áreas de las matemáticas como el álgebra y la geometría (Segerman, 2016), y da la posibilidad de manipular los modelos creados una vez impresos (Sun y Li, 2017).

Su inclusión en actividades y proyectos educativos interdisciplinares influye de manera positiva en las habilidades matemáticas de los alumnos, y en la motivación de alumnos y docentes (Berry et al., 2010; Huleihil, 2017; Stansell y Tyler-Wood, 2016). En estos proyectos, los estudiantes "tienen que usar medidas y cálculos matemáticos exactos, razonamiento científico en sus diseños, y tecnología para convertir sus ideas en soluciones reales" (Stansell y Tyler-Wood, 2016, p. 484).

Este recurso se presta muy bien a utilizarse de manera colaborativa, creando experiencias activas donde los alumnos aprenden a analizar y construir modelos tridimensionales por medio del ensayo y error, pudiendo validar y refinar el proceso de creación desarrollando la competencia de modelización matemática (Niss, 2003).

Por otro lado, la posibilidad de implantar la tecnología de impresión 3D a bajo coste dota a esta herramienta de una gran versatilidad y permite su uso también como herramienta educativa en entornos no formales (Canessa et al., 2013), como pueden ser los museos (Hart, 2005; Rainone et al., 2014) o los clubes de matemáticas que nos ocupan en este trabajo.

\section{Los clubes de matemáticas y el potencial matemático}

Desde sus primeras experiencias en Rusia a principios del siglo XX y en mayor medida desde su implantación en EEUU hacia finales del mismo siglo, los clubes de matemáticas (también Ilamados círculos matemáticos) se han extendido de manera internacional y se han convertido en soporte y complemento de los programas educativos de matemáticas de diferentes formas (Papanastasiou y Bottinger, 2004):

- Son lugares donde dar cabida a actividades de tipo exploratorio que tienen una menor presencia en el currículo regular. 
- Sus participantes se benefician tanto a nivel educativo como a nivel emocional, ya que participan en un ambiente más relajado y social fuera del horario de clases.

- Dan un apoyo a los alumnos en los problemas que encuentran en el currículo ordinario, creando un enlace entre las matemáticas de dentro y fuera de la escuela.

- Dan la oportunidad de desarrollar una actitud curiosa e investigadora ante las matemáticas, desarrolla su autoestima y les permite aprender de manera colaborativa, valorando el trabajo en equipo.

Otro aspecto destacable es que en algunos casos estos clubes consiguen el objetivo de mejorar las habilidades y actitudes de las niñas hacia asignaturas como las matemáticas, la informática y la ciencia en general (Karp y Niemi, 2000).

En muchas ocasiones los clubes de matemáticas acogen a estudiantes con buen rendimiento en matemáticas. En estos casos su objetivo es dar la oportunidad a estos alumnos de aprender contenidos que van más allá del currículo regular junto con otros alumnos con similares capacidades. Por otro lado, los clubes que incluyen alumnos con distintos niveles de habilidad matemática siempre tienen en común un alto interés en participar en actividades matemáticas fuera del horario de clase (Prescott y Pressick-Kilborn, 2015).

Los resultados del estudio de Papanastasiou y Bottinger (2004) mostraron actitudes muy positivas de los estudiantes acerca de las matemáticas y el club. Añaden, además, que "como las actitudes positivas están asociadas con niveles altos de rendimiento matemático, estos clubes tienen el potencial de incentivar a los estudiantes a participar en clases de matemáticas adicionales durante su educación secundaria, así como cursar estudios universitarios relacionados con las matemáticas" (p. 159). Es en este sentido en el que podemos hablar de los clubes de matemáticas como espacios de aprendizaje en donde los alumnos pueden desarrollar su potencial matemático.

En relación con las altas capacidades, Singer (2018) incluye la participación en clubes de matemáticas entre una de las actividades cruciales para desarrollar las habilidades matemáticas de estos estudiantes, junto con los programas especializados en altas capacidades, el apoyo familiar en el desarrollo de su talento y la colaboración entre padres y escuela. De hecho, Singer et al. (2016) resaltan cómo los programas extra-curriculares en algunos casos sirven como complemento y enriquecimiento de los programas reglados de atención a las altas capacidades, y en otros casos son el único espacio en donde detectar, estimular y realzar la alta capacidad matemática. En concreto, en "actividades como clubes de matemáticas, competiciones, cursos online, aprendizajes basados en proyectos y el trabajo con mentores dentro y fuera de la escuela, (...) los métodos de enseñanza y los retos matemáticos que se plantean en estos entornos son importantes para fomentar el talento matemático" (p. 24).

\section{El Club de Matemáticas de la Scuola Italiana de Madrid}

El Club de Matemáticas de la Scuola Italiana de Madrid se pone en marcha en el curso 2015-2016, y nace siguiendo el ejemplo de otros clubes que existen interna- 
cionalmente y que han tenido éxito: el Prime Factor Math Circle and School of Programming de Seattle (EUA) ${ }^{1}$, el Berkley Math Circle (for elementary school students) ${ }^{2}$ de Berkley (EUA), y el The Math Circle ${ }^{3}$ de Harvard (EUA), solo por citar algunos. En España, una experiencia similar se recoge en Callejo de la Vega (2010).

Su motivación y objetivo primordial es el de "desarrollar el pensamiento, o bien el razonamiento, lógico, matemático, deductivo" (Madonna y Park, 2017, p. 7). El primer curso del club se realizó con dos grupos distintos de alumnos de $3^{\circ}$ de Primaria (Grupo 1), el primero de 14 alumnos y el segundo de 5 alumnos, con los que se realizaron las mismas 10 sesiones con cada uno. La descripción de esta experiencia inicial se recoge en (Madonna y Park, 2017). En los años posteriores se realizaron 30 sesiones durante todo el año con tres grupos estables de distintas edades que se fueron creando durante este periodo, como se puede ver en la Tabla 1.

Tabla 1. Grupos y participantes del club desde el curso 2015-2016

\begin{tabular}{|c|c|c|c|c|c|c|c|}
\hline Curso & Grupo 1 & & Grupo 2 & & Grupo 3 & & Total Alumnos \\
\hline $2015-2016$ & $3^{\circ}$ Primaria & $14+5$ & & & & & 19 \\
\hline $2016-2017$ & $4^{\circ}$ Primaria & 17 & $6^{\circ}$ Primaria & 8 & & & 25 \\
\hline $2017-2018$ & $5^{\circ}$ Primaria & 13 & $1^{\circ}$ ESO & 6 & $3^{\circ}$ Primaria & 19 & 38 \\
\hline $2018-2019$ & $6^{\circ}$ Primaria & 11 & $2^{\circ}$ ESO & 5 & $4^{\circ}$ Primaria & 20 & 36 \\
\hline $2019-2020$ & $1^{\circ}$ ESO & 11 & $3^{\circ}$ ESO & 3 & $5^{\circ}$ Primaria & 12 & 26 \\
\hline $2020-2021$ & $2^{\circ}$ ESO & 4 & & & $6^{\circ}$ Primaria & 9 & 13 \\
\hline
\end{tabular}

El Grupo 1 que empezó en $3^{\circ}$ Primaria se mantuvo estable durante los siguientes años hasta $2^{\circ}$ de ESO, en donde se ha apreciado un importante descenso en el número de alumnos coincidiendo con el cambio a actividades online debido a la actual pandemia. Por el contrario, la mayor parte del Grupo 3 que se creó en el curso 20172018, que actualmente se encuentran en el último curso de Primaria, sí ha continuado con las actividades on-line. Han sido poco frecuentes los casos de alumnos que se han incorporado nuevos después de la formación inicial de los grupos, solamente existiendo un ligero descenso anual. El Grupo 2 de $6^{\circ}$ de Primaria que se creó en el curso 2016-2017 estaba constituido por los hermanos de los alumnos del Grupo 1 de primaria del año anterior.

Las clases se desarrollan los viernes por la tarde, después del horario de clases de la escuela, con una duración de una hora y media. No existe un método de selección de los alumnos, siendo una de las actividades extra-escolares ofertadas por el colegio, sin relación directa con el rendimiento escolar. El perfil de los alumnos, en general, es de alta motivación y potencialidad de cara a las matemáticas. Son alumnos que muestran entusiasmo tanto por las matemáticas escolares como por las actividades y

1. Prime Factor Math Circle and School of Programming: http://pfmathcircle.org/

2. Berkley Math Circle: http://mathcircle.berkeley.edu/

3. The Math Circle de Harvard: http://www.themathcircle.org/ 
encuentros del club. No podemos afirmar que algunos de los alumnos posean altas capacidades dado que este hecho no ha sido comprobado. Sin embargo, el tener un horario tan particular y el tratar unas actividades con características extracurriculares en muchos casos avanzadas, han ido seleccionando de manera indirecta a los alumnos consiguiendo unos grupos con perfiles de alta potencialidad matemática.

Las actividades principales de las sesiones del club están centradas en la resolución de problemas en pequeños grupos. Estas sesiones se alternan con otras de carácter más recreativo, como juegos matemáticos, ajedrez o papiroflexia entre otros, con la intención de mostrar a los alumnos aspectos más lúdicos de las matemáticas. Además, el club también promueve actividades y proyectos transversales para mostrar la presencia y utilidad de las matemáticas en otras disciplinas como las experiencias con la impresora 3D y el aeromodelismo que se describen en el presente trabajo.

\section{Método}

Este trabajo sigue una metodología de carácter descriptivo y exploratorio. A partir de las experiencias de aula llevadas a cabo con la impresión 3D durante los cursos 2017-2018, 2018-2019 y 2019-2020, se realiza un análisis y discusión de las producciones de los alumnos. En base a esas producciones se distinguen cuatro tipos de actividades en donde los alumnos se sintieron más involucrados y obtuvieron resultados más satisfactorios.

La experiencia se realizó con dos grupos del Club Matemáticas de Madrid de la Scuola Italiana. Comenzó como parte del proyecto de "Aeromodelismo y Matemáticas" en el curso 2017-2018 con el Grupo 1 de $5^{\circ}$ de Primaria (13 alumnos), y se repitió la misma actividad en el curso 2018-2019 con el Grupo 3 de $4^{\circ}$ de Primaria (20 alumnos). El proyecto "Aeromodelismo y Matemáticas" incluía la impresión 3D de partes de un aeroplano, lo que ayudó a iniciar el trabajo en el club con esta herramienta. En el curso 2018-2019 el Grupo 1 de 6º de Primaria (11 alumnos) trabajó con la impresora 3D en momentos puntuales. Durante el curso 2019-2020 el trabajo con la impresora 3D lo realizaron los Grupos 1 y 2 de forma conjunta (en total 14 alumnos). El número de alumnos y los distintos grupos se encuentran en la Tabla 1.

Las clases de impresión 3D se desarrollaron con la impresora Delta WASP 2040 en el Aula de Laboratorio de la Scuola Italiana. El software de diseño 3D que se utilizó fue TinkerCad, ${ }^{4}$ ya que además de ser libre y tener un manejo online sencillo e intuitivo, posee una amplia comunidad educativa (incluye un amplio repositorio de modelos y tutoriales) y permite la creación de Classrooms donde poder compartir los modelos entre los alumnos y el profesor. Cabe resaltar la utilidad de esta herramienta ya que el profesor puede hacer un seguimiento continuo del trabajo que están realizando los alumnos.

El software de laminado utilizado para la impresión fue Cura dada su eficiente compatibilidad con la impresora.

El análisis de producciones está basado principalmente en los distintos modelos tridimensionales creados por los alumnos en TinkerCad y los resultados obtenidos de sus impresiones 3D.

\footnotetext{
4. TinkerCad: https://www.tinkercad.com
} 


\section{Desarrollo de la experiencia}

Comenzamos con la descripción del proyecto "Aeromodelismo y Matemáticas" en donde tuvo lugar el primer contacto de los alumnos del club con la impresión 3D. El aeromodelismo proporcionó un campo en el que las matemáticas se presentan aplicadas a un contexto real. La modelización de objetos físicos concretos, como son los aeroplanos, y el uso de datos reales con herramientas digitales permiten experiencias muy motivadoras en los estudiantes de matemáticas (Morante y Vallejo, 2011).

La supervisión de las actividades fue llevada a cabo por el mismo docente. Las clases combinaban una parte teórica y una práctica en donde se construyeron una serie de aeroplanos y se aprendió el manejo de un simulador de vuelo. Los modelos no incluían instrucciones de montaje, solamente se proporcionaba una imagen del conjunto ya montado. Esto hizo que los estudiantes tuvieran que: (1) observar muy bien las piezas y su función con relación al conjunto; (2) intentar construir la secuencia de montaje antes de realizarla, con la idea de simular mentalmente los pasos; (3) documentar los pasos y las herramientas necesarias para cada paso, y (4) proceder al montaje y verificar si se correspondía con el resultado esperado y eventualmente corregir los errores.

El simulador (libre) de vuelo utilizado fue $R / C$ Desk Pilot ${ }^{5}$ y sirvió como preparación para una salida de campo que se realizó en un fin de semana. En ella los estudiantes realizaron varias sesiones de vuelo teledirigido bajo la supervisión y asistencia de personal cualificado. El objetivo fue probar en la realidad los aviones construidos en clase, además de vivir la emoción del vuelo teledirigido con algo concebido por los propios estudiantes.

Como culminación del proyecto de aeromodelismo se propuso a los alumnos imprimir piezas para alguno de los aviones que habíamos utilizado, dando de esta forma un sentido práctico y de gran expectación a la introducción de la impresora 3D del laboratorio. Después de una sesión colectiva el grupo estableció como objetivos imprimir los siguientes objetos:

1. Un monedero o portamonedas (ver Figura 1).

2. Un dron (a partir de modelos obtenidos del repositorio Thingiverse ${ }^{6}$ ).

3. Unas piezas del ala de uno de los aviones que se habían roto durante las prácticas (ver Figura 2).

El primer paso fue aprender a manejar TinkerCad, conocer sus funcionalidades básicas y sus distintas vistas, movimientos y rotaciones, elementos básicos como cubos, cilindros, esferas o conos (en la Figura 3 pueden verse alumnos del club utilizando el software). A continuación, se pasó a realizar en grupos la construcción de las primeras piezas de aprendizaje: un lápiz, una taza, varias piezas de ajedrez y un spinner, que permitieron a los alumnos conseguir un manejo autónomo del software.

Tras esta primera experiencia, en los años siguientes se continuó con el trabajo de diseño e impresión 3D en el club. Durante el curso 2018-2019 el Grupo 3 de $4^{\circ}$ de Primaria realizó el mismo proyecto de "Aeromodelismo y Matemáticas" repitiendo

5. R/C Desk Pilot: https://rcdeskpilot.com

6. Thingiverse: http://www.thingiverse.com 
las actividades realizadas el año anterior por el otro grupo, y el Grupo 1 de $6^{\circ}$ de Primaria realizó sesiones de impresión 3D libres. Ambas actividades fueron conducidas por el mismo docente.

Figura 1. Dos de los diseños de monederos de euros por parte de los alumnos

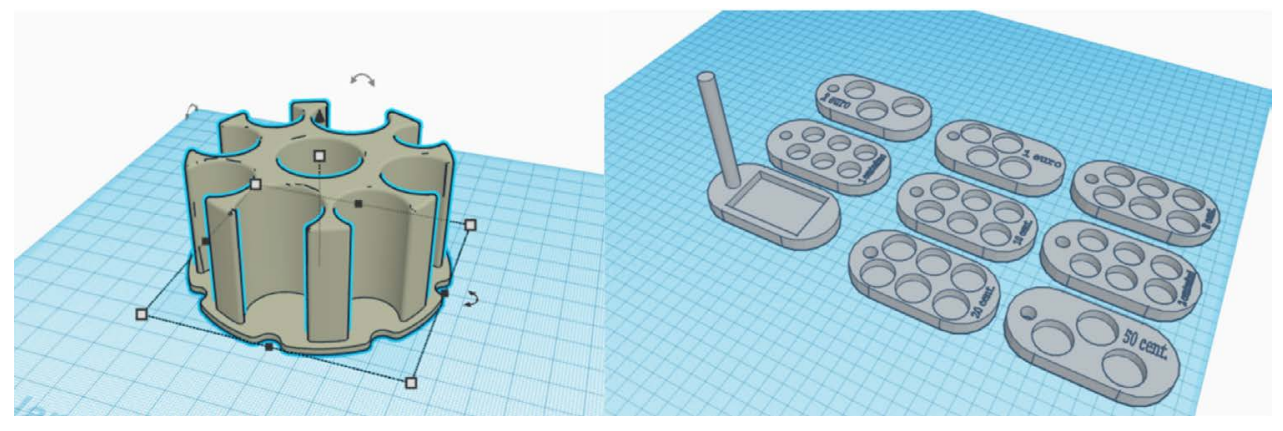

Figura 2. Diseño en TinkerCad y colocación final de las piezas del ala del avión
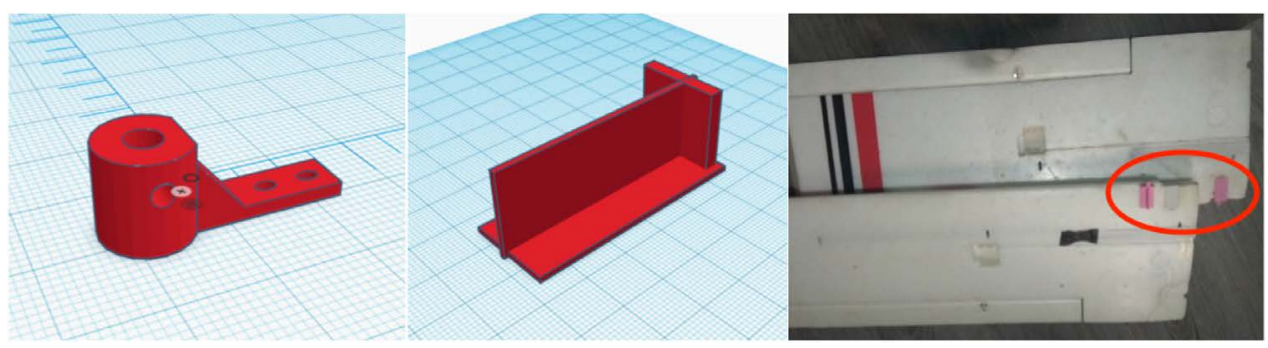

Figura 3. Alumnos del Club utilizando el software TinkerCad

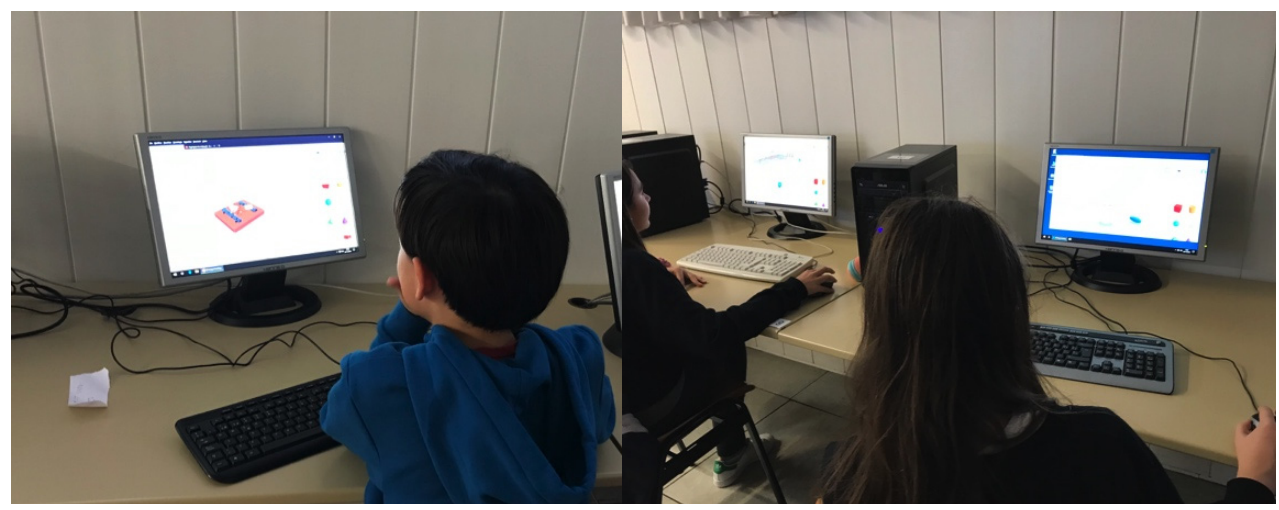


En los meses de noviembre y diciembre del curso 2019-2020 se continuó el trabajo con la impresora 3D durante cinco sesiones con los Grupos 1 y 2, formado por 11 alumnos de $1^{\circ}$ ESO y 3 alumnos de $3^{\circ}$ de ESO. Todas las actividades realizadas fueron supervisadas por dos docentes. Además de continuar con el trabajo de diseño y modelización tridimensional, el objetivo de estas sesiones fue que los estudiantes profundizaran en las fases del proceso de impresión 3D. Estas fases son las siguientes:

Fase 1. Diseño del objeto a imprimir con un software de modelado 3D (en nuestro caso TinkerCad) y creación del archivo .stl con el modelo tridimensional.

Fase 2. Proceso de laminado (slice) del objeto 3D con un software de laminado (en nuestro caso Cura) que traduce la información tridimensional del objeto geométrico en un archivo .gcode admitido por la impresora con todos los parámetros necesarios (número y grosor de las capas, relleno, soportes, etc).

Fase 3. Impresión del objeto y evaluación del resultado por si fuese necesario volver a la fase de diseño.

En la primera sesión de las 5 realizadas en el curso 2019-2020, se explicaron las 3 fases del proceso de impresión 3D y utilizando la pantalla digital del aula se diseñó de manera conjunta un modelo sencillo con TinkerCad. Se mostró al grupo de manera breve el funcionamiento del laminador Cura, haciendo que los estudiantes modificaran algunos parámetros de grosor de lámina y porcentaje de relleno en el interior del objeto. Mientras se imprimía el objeto, los alumnos comenzaron a realizar alguna de las lecciones de aprendizaje guiado de la propia plataforma para recordar el manejo del software.

En las siguientes sesiones de este último curso, se dejó la libertad a los alumnos de formar grupos y elegir los objetos y modelos que querían crear y posteriormente imprimir. El grupo de docentes animó a los estudiantes a formar grupos y a realizar construcciones o estructuras conjuntas, haciendo hincapié en la importancia de tener un proyecto o meta final a la que dirigir sus ideas.

\section{Resultados}

Tras las experiencias llevadas a cabo, queremos destacar 4 tipos de proyectos que surgieron a partir del trabajo de los alumnos durante las distintas sesiones, tanto por los contenidos y procesos matemáticos que pusieron en marcha como por el interesante trabajo colaborativo al que dieron lugar:

1. Objeto común con múltiples diseños. El objetivo es diseñar un objeto de uso cotidiano, cercano a los estudiantes y que existan diferentes maneras de diseñarlo. Por ejemplo, un monedero o un Ilavero.

2. Objeto diseñado para una situación real específica. Se plantea un problema y queremos diseñar un objeto que resuelva dicho problema. Por ejemplo, una pieza de repuesto o un soporte.

3. Proyecto integrado. Cada alumno diseña un elemento en un proyecto a mayor escala. Por ejemplo, el diseño de los edificios de una maqueta de una ciudad, o una calle o habitación con sus elementos. 
4. Diseños modulares. El objetivo es diseñar un único objeto, en principio complejo, que puede dividirse en distintas partes o módulos. Los módulos podrían asignarse a distintos alumnos y se agruparían para formar una estructura completa, por ejemplo, un robot. O bien, el objeto puede estar constituido por módulos iguales (o similares) que se ensamblan para formar el objeto final, como puede ser un mosaico o una construcción tipo Lego.

Una característica común a estos 4 tipos de actividades es que siempre involucraron a varios de los alumnos o grupos de alumnos, fomentando el trabajo colaborativo.

En los distintos periodos de trabajo con el diseño 3D y la impresora, los alumnos tuvieron la oportunidad de diseñar varios objetos con TinkerCad, algunos dentro de las cuatro categorías anteriores y otros no. A continuación, analizaremos un ejemplo de cada uno de los anteriores cuatro tipos de proyectos.

Ejemplo 1. El monedero de euros (objeto común de múltiples diseños; Grupo 3, curso 2018-2019)

Algo tan sencillo como un monedero de euros se convirtió en un interesante ejercicio colaborativo de modelización. Después de una puesta en común de ideas, cada uno de los grupos eligió una forma diferente de representar un monedero. La diferencia entre los distintos modelos que surgieron a raíz de esta tarea (ver Figura 2) puso de manifiesto el componente de creatividad en un proceso abierto de modelización.

De especial interés desde el punto de vista de los contenidos matemáticos desarrollados en este ejemplo fue el trabajo de medida realizado para que los valores reales de cada moneda fuesen representados de manera correcta tanto en el modelo 3D de TinkerCad como en su posterior impresión. A partir de monedas reales se realizó un trabajo de estimación del radio y grosor de las distintas monedas, que involucró el problema de la determinación de sus centros. Aparecieron distintas estrategias de resolución de este problema, como calcar la circunferencia en un papel y doblarla en dos mitades, o calcular el circuncentro de un triángulo inscrito cualquiera. En los procesos de impresión se pudo comprobar si las medidas fueron las correctas, e incluso se llegó a imprimir alguna moneda para compararla con la real.

Ejemplo 2. Piezas del ala de un aeroplano (objeto diseñado para una situación real específica; Grupos 1 y 3, cursos 2017-2018, 2018-2019)

El diseño de las piezas del ala de uno de los aviones se presentó como una excelente oportunidad de enlazar el trabajo de diseño 3D con TinkerCad con el proyecto de aeromodelismo. Además, se consiguió relacionar de manera directa el uso de la tecnología de impresión 3D con un problema práctico, aplicado a un contexto real y cercano a los estudiantes. En la Figura 3 se puede ver el diseño en TinkerCad de las dos piezas y su colocación en las alas del avión.

Las piezas no tenían un diseño muy complejo y fueron obtenidas por el conjunto de alumnos sin muchos problemas. Desde el punto de vista matemático destacó la necesidad de trabajar con medidas reales muy precisas, forzando a que se tuviera que imprimir la pieza y comprobar su viabilidad varias veces hasta encontrar el modelo óptimo. Este proceso de ensayo y error permitió a los alumnos recorrer varios ciclos de modelización matemática. 
Por otro lado, surgió el factor del peso de las piezas una vez impresas, aspecto importante a la hora de poder ser utilizadas para que el avión mantenga la estabilidad en el vuelo. En este punto los parámetros de impresión y la densidad del filamento jugaron un papel importante a la hora de conseguir la pieza final.

Ejemplo 3. Los "Edificios Famosos" (proyecto integrado; Grupo 1, curso 2019-2020)

En uno de los grupos varios alumnos desarrollaron modelos de distintos edificios emblemáticos, como la Torre de Pisa, el Empire State o la Torre Eiffel (ver Figura 4). A partir de las medidas reales e información que encontraron en internet crearon modelos de estos edificios. Partiendo de una primera versión muy simplificada, fueron incluyendo poco a poco detalles cada vez más realistas, evaluando en cada paso con la ayuda de imágenes de los edificios si su modelo coincidía con el real y realizando las modificaciones necesarias para adaptarlo.

Figura 4. Diseño en TinkerCad e impresión 3D del Empire State y la Torre Eiffel
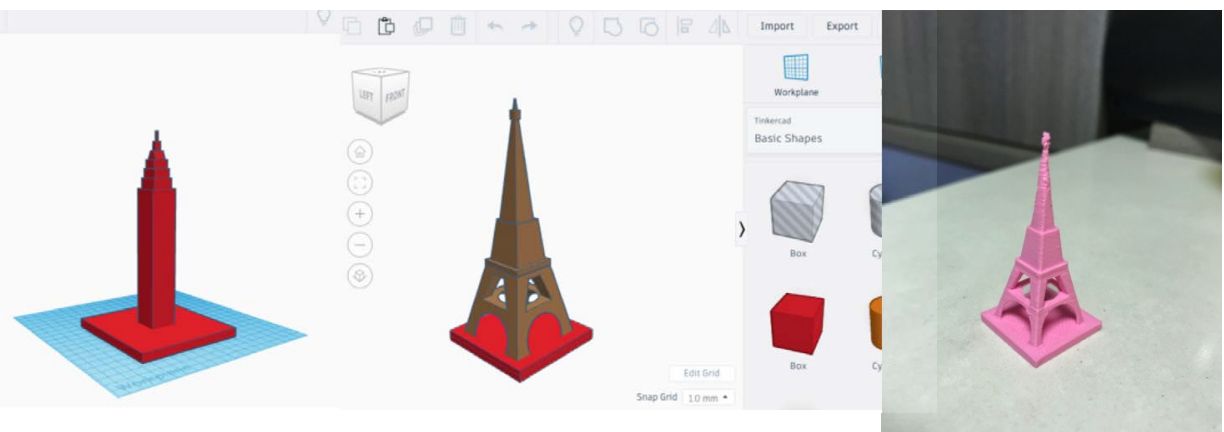

Entre los aspectos matemáticos involucrados en los diseños de los edificios destacamos el manejo de las proporciones entre el modelo creado y la realidad de los distintos elementos de las torres (zonas de ventanas, tramos uniformes diferenciados, altura de los arcos, altura de las agujas, etc.) y el diseño de cada una de estas partes mediante la unión e intersección de sólidos para afinar detalles.

Por otro lado, con la impresión de los dos primeros edificios, la Torre Eiffel y el Empire State, se pudo comprobar que la escala no era la adecuada ya que ambos modelos se imprimieron con el mismo tamaño. Como en la realidad el Empire State es 80 metros más alto que la torre parisina, los alumnos tuvieron que reajustar la escala de sus modelos para adecuarse a esta diferencia. Es decir, al considerar a los edificios dentro de un proyecto integrado el trabajo relacionado con la escala cobra mayor peso.

Al final, se pensó en continuar con este proyecto integrado planificando la maqueta de una ciudad imaginaria en donde estuvieran todos los edificios colocados, además de incluir otros elementos urbanos. El desarrollo de esta maqueta hubiera involucrado un trabajo de proporción y escala más ambicioso que por falta de tiempo no se pudo completar. 
Ejemplo 4. El tanque (diseño modular; Grupo 3, curso 2019-2020)

El grupo de 3 alumnos de $3^{\circ}$ de ESO durante el curso 2019-2020 decidió crear un tanque. Después de consultar imágenes de tanques por internet eligieron el modelo en el que se iban a basar para desarrollar el suyo propio, y decidieron dividir el tanque en tres partes para que cada uno de ellos diseñara una de ellas: base y ruedas, cuerpo y cañón. Comenzaron a trabajar primeramente de manera individual, manteniendo siempre una comunicación constante para que las medidas de todas las piezas fueran las mismas. El trabajo "en papel" fue también muy significativo, poniendo en común la estructura final del tanque y la manera en que cada pieza se ensamblaba para formar el objeto final (ver Figura 5).

Figura 5. Diseño en TinkerCad e impresión 3D del tanque

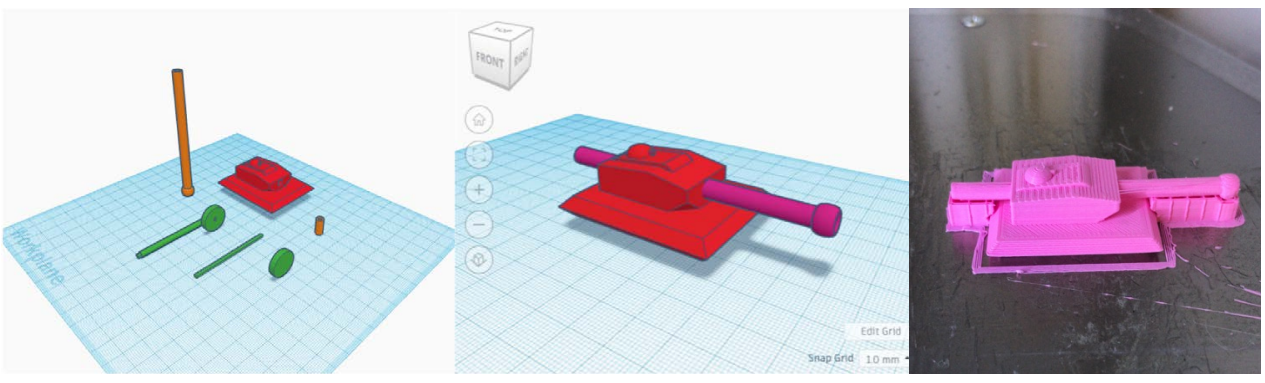

En este mini-proyecto, los aspectos matemáticos más destacables son el de la descomposición de una forma compleja en formas más sencillas y la identificación de figuras geométricas que pudieran modelizar la figura. Por otro lado, la labor de comunicación para mantener la proporcionalidad en el resultado final, así como la toma de decisiones para conseguir que las piezas encajaran entre sí fue significativo.

Cabe destacar también la calidad en el detalle en cada una de las partes que elaboraron, trabajando de forma concienzuda en la intersección de sólidos para conseguir los perfiles buscados. Finalmente, en lugar de realizar una impresión por módulos, utilizaron el aula de TinkerCad para ensamblar las distintas piezas en un único modelo, verificando el correcto diseño de cada uno de los componentes.

\section{Discusión}

En todo momento los alumnos mostraron un gran interés y participación en las sesiones de impresión 3D del club. En algunas ocasiones los alumnos optaron por comenzar de manera individual con actividades de modelización más libre, no incluidas dentro de los cuatro tipos anteriores. En general sus modelos acababan en proyectos muy elaborados que dieron lugar a procesos complejos o fallidos de impresión. En este tipo de ejemplos encontramos una placa de YouTube, una cancha de baloncesto, el interior de iglesias y casas, un árbol de navidad o un parchís (Figura 6). Sin embargo, hubo ocasiones en donde estas iniciativas se completaban con éxito y conseguían atraer la atención de otros alumnos del club creando de manera espontá- 
nea una actividad de las cuatro mencionadas anteriormente. Es el caso, por ejemplo, de los Edificios Famosos, que surgió a partir de la creación de una alumna del Empire State y atrajo a dos de sus compañeras hacia un proyecto integrado de modelar otros edificios emblemáticos.

Figura 6. Diseño en TinkerCad e impresión 3D del parchís

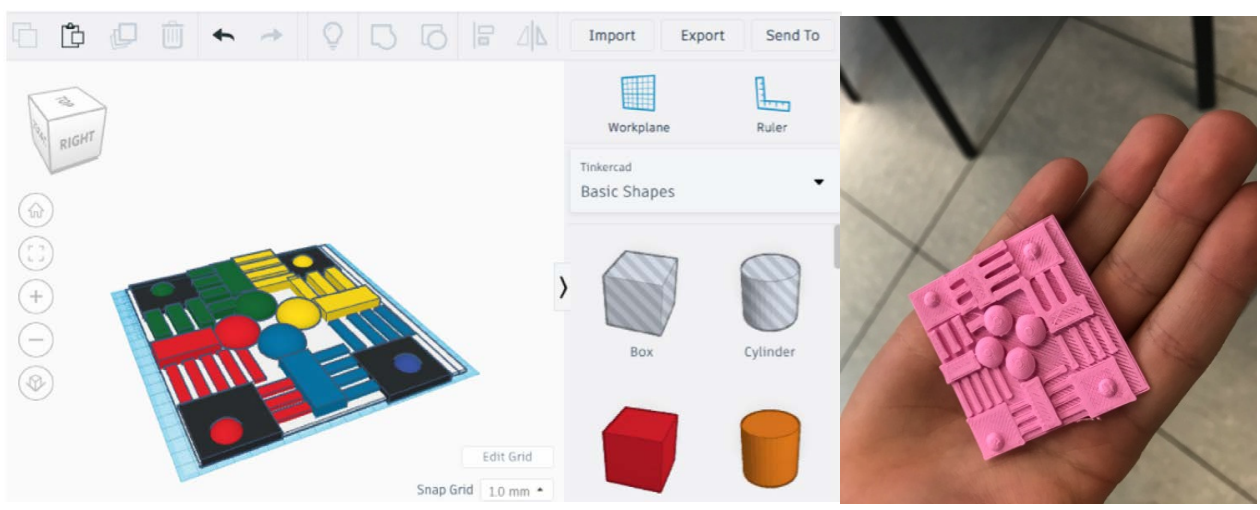

Cabe destacar que incluso cuando los resultados obtenidos por los alumnos no fueron los deseados, como el caso de modelos complejos o no imprimibles, los alumnos siguieron mostrando interés en las tareas de modelización. A pesar de la ausencia de éxito en estos casos, tuvieron una buena predisposición hacia la creación de nuevos modelos en TinkerCad, comenzando a continuación un nuevo proceso de modelado que dotó a los alumnos de un alto grado de manejo con esta herramienta. A su vez, cuando la impresión 3D producía resultados inesperados, se aprovechó para conocer las limitaciones del proceso de impresión y entender qué modelos o estructuras tienen una mayor probabilidad de ser impresos con éxito, ayudando así en los procesos de rediseño.

En relación con las limitaciones de la tecnología de impresión 3D, destacamos las siguientes observaciones:

- El tiempo de impresión es muy largo y las piezas deben dejarse varias horas para ser imprimidas con lo que el resultado no es inmediato. Aunque la mayoría no conseguía ver su modelo impreso, al tenerlo al comienzo de la siguiente sesión les motivaba a modificar ese o diseñar uno nuevo.

- Uso de los soportes. Para imprimir las partes que sobresalen en los modelos la impresora necesita imprimir soportes que deben quitarse una vez finalizada la impresión. Los alumnos comprobaron la importancia de colocar adecuadamente los modelos y tener en cuenta que figuras con muchos detalles necesitan mucho soporte lo que complicará su realización final (no es fácil "eliminar" los soportes).

- Algunos alumnos comenzaban estructuras muy complejas y con demasiados elementos que requería de una alta precisión. Por ejemplo, un estudiante comenzó a diseñar una casa con todos los elementos de su interior (habitaciones, 
muebles, etc.), que finalmente desechó por intentar llegar al máximo detalle. Después de entender la dificultad de imprimir ese tipo de modelos, acabó desconectándose del proceso de impresión 3D y dedicando el tiempo a modelar distintos objetos en TinkerCad. Este alumno entró dentro de un grupo pequeño de alumnos que mostró más interés en las tareas de modelado 3D que en la obtención del resultado final impreso.

- Problemas de escala. Los diseños que ideaban originalmente algunos alumnos no se correspondían con lo que luego podía imprimirse en un tiempo razonable. Por ejemplo, en el parchís de la Figura 6 el alumno comprobó que no se podía jugar con fichas reales y considerando el tiempo que se tardaría en imprimir un tablero grande abandonó la idea. Este alumno, además, observó la diferencia entre los colores usados en el software y el único color impreso.

- Objetos difíciles de imprimir. Uno de los grupos diseñó una cancha de baloncesto, en donde los jugadores y la canasta no se conseguían imprimir satisfactoriamente por ser demasiado finos o con muy poco soporte. En estos casos el proceso de rediseño e impresión debe alargarse hasta alcanzar un resultado óptimo. Sin embargo, al ser los tiempos de impresión tan largos los alumnos perdían el interés en seguir modificando el modelo y prefirieron comenzar a modelar uno nuevo.

En relación al aprendizaje sobre las fases del proceso de impresión, la mayor parte de los alumnos experimentaron con la Fase 1 (diseño 3D) y Fase 3 (impresión 3D). En la Fase 1 se pudo apreciar una gran mejora en el manejo del software de diseño 3D TinkerCad y mostraron mucho interés en conocer sus posibilidades. En varias ocasiones recurrieron a los tutoriales de TinkerCad para poder incluir distintas construcciones en sus modelos o realizar alguna operación con los cuerpos geométricos (intersección, unión o sustracción), incrementando así el conocimiento que tenían sobre el manejo del software. En relación con la Fase 3 los alumnos pudieron comprobar las características de esta forma de impresión por capas y reconocer algunas de sus limitaciones, como puede ser el tiempo de impresión.

Aparte de las explicaciones teóricas iniciales sobre el proceso de la impresión $3 \mathrm{D}$, la Fase 2 fue realmente experimentada únicamente con el grupo de $3^{\circ}$ de la ESO, en particular con el ejemplo de tanque. Este grupo fue el que mostró más interés en conocer el funcionamiento del software de laminado Cura y de experimentar con los distintos parámetros que permite modificar (grosor y tamaño de cada capa, porcentaje de relleno, tipo de soporte, etc.). El mayor conocimiento de estos alumnos puede explicar este mayor interés en esta parte del proceso.

\section{Conclusiones}

La experiencia en el desarrollo de estas actividades con los estudiantes del club ha sido muy positiva. La conexión del aeromodelismo con la impresión 3D supuso una oportunidad de culminar el proyecto transversal que generó un interés inesperado en los estudiantes sobre el potencial creativo de la impresión 3D. Incluir esta tecnología en las aulas de matemáticas dota a las actividades realizadas de un sentido práctico de gran valor, además de despertar la motivación y curiosidad de los alumnos. 
La introducción de la impresión 3D en las aulas está en fase de experimentación, así como el tipo de actividades que se pueden trabajar con los alumnos para obtener que sea un apoyo para el aprendizaje de conceptos matemáticos. A partir de las actividades desarrolladas en el club con la impresora 3D hemos podido distinguir cuatro tipos de proyectos de modelado 3D: objeto común con múltiples diseños, objeto diseñado para una situación real específica, proyecto integrado y diseños modulares, en donde los alumnos se han visto más involucrados y donde el trabajo realizado ha sido más completo. En ellos, los alumnos participaron de manera colaborativa y activaron un mayor número de procesos matemáticos relacionados con el diseño y la modelización. Es evidente que un alumno que diseña con una herramienta tecnológica la Torre Eiffel ha resuelto un problema de modelización, pero trabaja más competencias si tiene que colocar la torre en la maqueta de una ciudad con otros compañeros. Necesita ajustar la escala de la torre con los edificios cercanos, conectar e interpretar su modelo en relación con el contexto urbano de la maqueta, comunicar y colaborar para completar el proyecto.

En la experiencia desarrollada, estos cuatro tipos de proyectos crearon más interacción entre los alumnos y consiguieron alcanzar impresiones más satisfactorias frente a los proyectos con un carácter más libre e individual, que en ocasiones finalizaban con modelos demasiado complejos o no imprimibles. Su carácter colaborativo hizo que los alumnos se involucraran con mayor interés en la resolución de los problemas matemáticos de proporción, escala y operaciones con sólidos que se les planteaban en el proceso de diseño e impresión de los modelos.

El perfil de estudiantes de los clubes de matemáticas cuenta con alumnos con buena predisposición a trabajar en problemas de matemáticas, además de estudiantes con alto potencial o altas capacidades. La inclusión de la impresión 3D como recurso crea un entorno motivador para este tipo de alumnado, a la vez que propone diversos retos y problemas matemáticos surgidos de las tareas de modelización y diseño tridimensional que son requeridas. El trabajo con esta herramienta da a estos alumnos la oportunidad de profundizar en aspectos transversales de una manera novedosa, sirviendo como complemento en el desarrollo de su potencial matemático.

La distinción entre los tipos de proyectos realizables con la impresión 3D en un aula de matemáticas incluida en el presente trabajo proporciona una línea de investigación para seguir profundizando en las posibilidades educativas de esta novedosa tecnología.

\section{REFERENCIAS BIBLIOGRÁFICAS}

Berry, R. Q., Bull, G., Browning, C., Thomas, C. D., Starkweather, G. y Aylor, J. (2010). Use of digital fabrication to incorporate engineering design principles in elementary mathematics education. Contemporary Issues Technology and Teacher Education, 10(2), 167-172.

Callejo de la Vega, M. L. (2010). Un club matemático para la diversidad. Narcea Ediciones.

Canessa, Ch. E., Fonda, C. y Zennaro, M. (Eds.) (2013). Low-Cost 3D Printing for Science, Education and Sustainable Development. ICTP Science Dissemination Unit. 
Department of Education (2013). 3D printers in schools: uses in the curriculum. Research and Analysis, October 2013, Gov.UK. https://www.gov.uk/government/ publications/3d-printers-in-schools-uses-in-the-curriculum

Ford, S. y Minshall, T. (2018). Where and how 3D printing is used in teaching and education. Additive manufacturing, 25, 131-150. https://doi.org/10.1016/j.addma.2018.10.028

Hart, G. W. (2005). Creating a mathematical museum on your desk. The Mathematical Intelligencer, 27, 14-17. https://doi.org/10.1007/BF02985853

Huleihil, M. (2017). 3D printing technology as innovative tool for math and geometry teaching applications. IOP Conf. Ser.: Mater. Sci. Eng., 164(1), 012023. http://doi. org/10.1088/1757-899X/164/1/012023

Karp, K. S. y Niemi, R. C. (2000). The math club for girls and other problem solvers. Mathematics Teaching in the Middle School, 5(7), 426-432.

Madonna, C. y Park, S.S. (2017). Como organizar un club de Matemática. Lulu.

Morante, A. y Vallejo, J. A. (2011). Modelling the landing of a plane in a calculus lab. International Journal of Mathematical Education in Science and Technology, 43(7), 936-949. https://doi.org/10.1080/0020739X.2011.633626

Niss, M. (2003). Mathematical competencies and the learning of mathematics: the Danish Kom Project. Roskilde University.

Papanastasiou, E. C. y Bottiger, L. (2004). Math clubs and their potentials: Making mathematics fun and exciting. A case study of a math club. International Journal of Mathematical Education in Science and Technology, 35(2), 159-171. https://doi. org/10.1080/00207390310001638395

Prescott, A. E. y Pressick-Kilborn, K. J. (2015). It's great to be doing maths! Engaging primary students in a lunchtime club. Australian Primary Mathematics Classroom, 20(3), 34-49.

Rainone, M. Fonda, C. y Canessa, E. (2014). IMAGINARY Math Exhibition Using Lowcost 3D Printers. ArXiv, abs/1409.5595.

Segerman, H. (2016). Visualizing Mathematics with 3D Printing. Johns Hopkins University Press.

Singer, F. M., Sheffield, L. J., Freiman, V. y Brandl, M. (2016). Research On and Activities For Mathematically Gifted Students. Springer Nature.

Singer F. M. (2018). Enhancing Creative Capacities in Mathematically-Promising Students. Challenges and Limits. En F. Singer (Ed.), Mathematical Creativity and Mathematical Giftedness (pp. 1-23). ICME-13 Monographs. Springer, Cham. https://doi.org/10.1007/978-3-319-73156-8_1

Stansell, A. y Tyler-Wood, T. (2016). Digital fabrication for STEM projects: a middle school example. IEEE 16th International Conference Advance Learning Technologies (ICALT), 483-485. https://doi.org/10.1109/ICALT.2016.44

Sun, Y. y Li, Q. (2017). The application of 3D printing in mathematics education. 12th International Conference on Computer Science and Education (ICCSE), 47-50. https://doi.org/10.1109/ICCSE.2017.8085461 